\title{
Autonomic dysfunction and peripheral nerve involvement in patients with
} Parkinson's disease

Daniela Matei $^{1}$, Catalina Luca ${ }^{1}$, Doru Andritoi ${ }^{1}$, Robert Fuior ${ }^{1}$, Dan Zaharia ${ }^{1}$, Ilie Onu ${ }^{1}$, Calin Corciova ${ }^{1}$

Corresponding author: Catalina Luca, E-mail: luca.katalina@yahoo.com,

${ }^{1}$ Department of Biomedical Sciences, Faculty of Medical Bioengineering, University of Medicine and Pharmacy "Grigore T. Popa" Iasi, Romania

\begin{abstract}
Introduction: Parkinson's disease (PD) is a chronic illness which damages central and peripheral nervous system. The presence of peripheral neuropathy (PN) in PD, it has been suggested to be the effect of treatment. The aim of this study was to investigate autonomic cardiac control in PD patients with normal serum levels of vitamin B12 by means of spectral analysis of short-term heart rate variability (HRV) and also to assess the prevalence of PN using electrophysiological examinations. Methods: 30 (18 male and 12 female) with PD were compared to 20 age- and sex-matched control subjects. Short-term ECG was used to calculate time domain and spectral parameters of HRV. The stimulodetection examination was realized in the motor fibers of median, peroneal and tibial nerves, and in the sensitive fibers of median and sural nerve according to the standard procedures. Results: Low and high frequency were lower in PD patients than in controls (LF: 332. $\pm 288.4 \mathrm{~ms}^{2}$ PD vs $723.9 \pm 348.2 \mathrm{~ms}^{2} \mathrm{C}$; HF: $283.72 \pm 241.97 \mathrm{~ms}^{2}$ PD vs $\left.530.54 \pm 226.5 \mathrm{~ms}^{2} \mathrm{C}, \mathrm{p}<0.01\right)$. No differences between LF/HF ratio of PD and controls appeared. Sensory nerve action potential in sural nerve was reduced in PD patients. No differences between sensory and motor nerve conduction velocities of PD and controls appeared. Conclusions: PD causes dysfunction of autonomic cardiovascular regulation and peripheral nerve involvement.
\end{abstract}

Key words: Parkinson's disease, heart rate variability, parasympathetic nervous system, low frequency, sensory nerve action potential, nerve conduction velocity,

\section{Introduction}

Parkinson's disease (PD) is a chronic neurodegenerative movement disorder characterized by a progressive loss of the pigmented dopaminergic neurons of the substantia nigra pars compacta ( $\mathrm{SNpc}$ ) and by the presence of intra-neuronal aggregates called Lewy bodies (1). This degeneration affects not only the dopaminergic mesocorticolimbic system, but also the noradrenergic locus coeruleus (2), motor vagal nucleus, the serotonergic raphe nuclei (3) and many peptidergic brainstem nuclei containing substance $\mathrm{P}$, somatostatin, and neuropeptide $\mathrm{Y}$ (4). Clinical manifestations of Parkinson's disease include motor dysfunction such as rigidity, resting tremor, bradykinesia, postural instability and gait dysfunction. PD is also associated with many nonmotor features, including autonomic dysfunction, pain, mood disorders, sleep impairment, and dementia (5).
The pathogenesis of PD is largely unknown but mitochondrial dysfunction, oxidative stress, glutamate induced excitotoxicity, intracellular protein accumulation of Lewy Bodies containing $\alpha$-synuclein protein, inflammation, loss of neurotrophic factors and apoptosis appear to have a key role in the development and progression of PD (6-8). Also the genetic factors, genes like parkin and $\alpha$-synuclein has been involved in pathogenesis of PD (9).

It is well documented that PD may be associated with a dysfunction of the autonomic nervous system. Histological studies have proven the presence of Lewi's bodies in sympathetic and parasympathetic preganglionic neurons and also in central structures associated with the autonomic regulation (10).

Analysis of heart rate variability (HRV) from ambulatory ECG recordings has become an important method for assessment of cardiovascular autonomic regulation. 
To characterize the cardiovascular autonomic regulation in PD, Ewing tests were used. These tests require an active participation of the patients, and the rigidity and tremor characteristic of PD make these tests difficult to assess. The measurement of autonomic parameters using power spectrum analysis of heart rate (HR) does not require the active participation of patients. Decrease of spectral power in PD patients was documented in 24-hour examination (11). The presence of peripheral neuropathy (PN) in PD is a relatively new clinical fact. PD patients may have sensory abnormalities which in some cases precede motor dysfunction. Alpha-synuclein was found in peripheral sensory neurons in PD patients (12). The presence of PN in $\mathrm{PD}$, it has been suggested to be an iatrogenic effect of levodopa treatment, by deficiency of vitamin B12 after treatment (13). Toth et al. found that high levels of methylmalonic acid (MMA) are associated with levodopa-treatment and PN in PD patients (14).

The aim of this study was to investigate autonomic cardiac control in PD patients in early stages of the disease, with normal serum levels of vitamin B12 (cobalamin) and methylmalonic acid, by means of spectral analysis of short-term HRV and also to assess the prevalence of PN using electrophysiological examinations.

\section{Metthods}

\section{Participant recruitment and inclusion criteria}

30 patients (18 male and 12 female) with PD were compared to 20 age- and sex-matched control subjects. Parkinson's disease was diagnosed according to the United Kingdom Parkinson Disease Society Brain Bank criteria (15). PD severity was determined using the Hoehn and Yahr scale (H\&Y) (16). The patients included into the study also meet the criteria of normal serum levels of vitamin B12 (cobalamin) and methylmalonic acid.

Patients with stroke, epilepsy, coronary artery disease, arrhythmias, atrioventricular block or bundle branch block were excluded from the study. Inclusion criteria for the controls were the absence of any history of PD and with two normal consecutive ECG in the course of one month. Patients who had medication known to influence autonomic system (anticholinergic and monoamine oxidase inhibitor) were excluded. The study was carried out in accordance with the Helsinki Declaration. All subjects participated voluntarily after being given a detailed explanation of the purpose of the study.

\section{Clinical and paraclinical assessment}

All the subjects underwent detailed history taking and neurological examination. A standardized autonomic questionnaire was used to detect autonomic complaints.

Score of Unified Parkinson's Disease Rating Scale (UPDRS) part III (Motor section) was recorded when patients were at "on" state (17). The motor examination of UPDRS is the most used measure to assess motor symptoms such as rest and action tremor, rigidity, bradykinesia, gait and posture, facial masking. Each items are scored on a 0 (no impairment) to 4 (severe impairment), the total motor UPDRS exam score ranges from 0 to 108 (17).

For each person in the study we measured: body mass index (BMI) (calculated as weight divided by height squared- $\left.K g / \mathrm{m}^{2}\right)$, fasting blood glucose, serum total cholesterol, high density lipoprotein cholesterol$H D L$, low density lipoprotein cholesterol-LDL, triglycerides; systolic and diastolic arterial blood pressures in standard examination conditions.

\section{Measurement of heart rate variability}

Using BIOPAC Acquisition System, we monitored the HRV in basal condition (18-20). HRV was analyzed following the recommendations of the Task Force of the European Society of Cardiology and the North American Society of Pacing and Electrophysiology (21). Short time ECG data were digitized and stored on computer for subsequent offline analysis. The ectopic bits or artifacts were manually edited.

Time-domain parameters used were Mean-R-R, Standard deviation of all NN intervals (SDNN), square root of the mean of the sum of the squares of differences between adjacent NN intervals (RMSSD), percentage of differences between adjacent $\mathrm{NN}$ intervals differing more than $50 \mathrm{msec}(\mathrm{pNN} 50 \%$ ). $\mathrm{pNN} 50 \%$ has been proven to be a valid index of vagal tone. Frequency Domain HRV measured were: very low frequency (VLF) from $0.0033-0.04 \mathrm{~Hz}$, low frequency (LF) from $0.04-0.15 \mathrm{~Hz}$ and high frequency (HF) $0.15-0.4 \mathrm{~Hz}$. The HF fluctuation of RR intervals mainly reflects the cardio-vagal modulation and the inspiratory inhibition of vagal tone (21). We analyzed LF and HF power, ratio of LF/HF (considered an index of cardiac sympathetic/parasympathetic tone balance). All HRV tests were made always in the morning hours between 9 and 11 a.m. 
Table 1. Recording and stimulation site for nerve conduction studies

\begin{tabular}{|c|c|c|c|}
\hline Nerve & $\begin{array}{c}\text { Recording } \\
\text { (surface electrodes) }\end{array}$ & Distal stimulation & $\begin{array}{c}\text { Proximal } \\
\text { stimulation }\end{array}$ \\
\hline $\begin{array}{c}\text { mNCV Median } \\
\text { nerve }\end{array}$ & $\begin{array}{l}\text { Abductor Pollicis } \\
\text { Brevis Muscle }\end{array}$ & $3 \mathrm{~cm}$ above the wrist & Elbow \\
\hline $\begin{array}{c}\text { mNCV Peroneal } \\
\text { nerve }\end{array}$ & $\begin{array}{l}\text { Extensor Digitorum } \\
\text { Brevis Muscle }\end{array}$ & $\begin{array}{l}\text { Ankle (between the extensor digitorum } \\
\text { longus and extensor hallucis longus } \\
\text { tendons) }\end{array}$ & $\begin{array}{l}\text { behind the fibular } \\
\text { head }\end{array}$ \\
\hline $\begin{array}{c}m \text { NCV Tibial } \\
\text { nerve }\end{array}$ & $\begin{array}{l}\text { Abductor Hallucis } \\
\text { Muscle }\end{array}$ & behind the medial malleous & popliteal fossa \\
\hline $\begin{array}{l}\text { sNCV Median } \\
\text { nerve } \\
\text { ortodromic } \\
\text { stimulation }\end{array}$ & $3 \mathrm{~cm}$ above the wrist & Finger II & \\
\hline $\begin{array}{l}\text { sNCV Sural nerve } \\
\text { antidromic } \\
\text { stimulation }\end{array}$ & $\begin{array}{l}\text { behind the Lateral } \\
\text { Malleolus }\end{array}$ & $\begin{array}{l}\text { distal to the lower border of the bellies of the } \\
\text { gastrocnemius, at the junction of the middle } \\
\text { and lower thirds of the leg, just lateral to the } \\
\text { midline. }\end{array}$ & \\
\hline
\end{tabular}

\section{Nerve conduction studies}

For electrophysiological investigations the electromyograph Neuro-MEP Micro was used. The stimulodetection examination was realized in the motor fibers of median, peroneal and tibial nerves, and in the sensitive fibers of median and sural nerve according to the standard procedures (22). The surface electrodes were used to obtain muscular response according to the principle "belly-tendon" (the active electrode was placed on the muscle belly and the reference electrode on its tendon). The ground was placed between distal stimulation and the recording electrodes. Stimulation and collection points are shown in Table 1. The investigations were carried out in a warm room, the temperature of the patient's skin being at least $35^{\circ} \mathrm{C}$. For data acquisition specific settings were used: sensitivity $(500 \mu \mathrm{V}-2$ $\mathrm{mV}$ for motor and $2-5 \mu \mathrm{V}$ for sensory nerve conduction velocity- $\mathrm{NCV})$; frequency range $(20 \mathrm{~Hz}$ - $5 \mathrm{kHz}$ motor NCV and $20 \mathrm{~Hz}-7 \mathrm{kHz}$ sensory $\mathrm{NCV}$ ); sweep $(5 \mathrm{~ms} /$ division motor and $2 \mathrm{~ms} /$ division sensory NCV). Stimulation used single stimuli with duration of $0.1 \mathrm{~ms}$ and variable intensity to obtain maximum response (maximum $100 \mathrm{~mA}$ ). For each motor nerve the following electro-physiological indices were studied: motor distal latency (MDL $\mathrm{ms}$ ), compound motor action potential (CMAP $\mathrm{mV}$ ), motor nerve conduction velocity ( $\mathrm{mNCV}$ $\mathrm{m} / \mathrm{s}$ ). At the examination of sensitive nerves the following parameters were measured: sensitive latency (SL - ms), sensory nerve action potential (SNAP - microV), sensory nerve conduction velocity $(\mathrm{sNCV}-\mathrm{m} / \mathrm{s})$.

\section{Statistical Analysis}

The statistical analysis of the results was performed using the software package STATISTICA 6.0 (StatSoft Inc., USA). The values are presented as mean values and standard deviation. Test t- Student or variance analysis (ANOVA) was used to determine the differences between the groups. The Pearson correlation coefficient $r$ was used for determining relationship between parameters. The values $p<0.05$ were considered statistically significant.

\section{Results}

We studied 30 patients (18 males and 12 females) with $\mathrm{PD}$ in $1-2 \mathrm{H} \& \mathrm{Y}$ stage (mean age $63.2 \pm 3.47$ years, body mass index $\left.24.44 \pm 1.74 \mathrm{~kg} / \mathrm{m}^{2}\right)$. These were compared to 20 normal patients (10 males/10 females, age $61.84 \pm 3.54$ years, BMI $25.63 \pm 1.56$ $\mathrm{kg} / \mathrm{m}^{2}$ ) without history of PD and cardiovascular event.

Characteristic of study groups, time and frequency domain parameters of HRV are summarizes in Table.2. UPDRS motor was $19.2 \pm 5.2$ in PD, orthostatic hypotension was found in 13 PD patients $(43.3 \%)$ and in 5 controls $(25 \%)$ patients with $p<0.01$. Mean systolic and diastolic blood pressure did not differ when compared to the control group, but heart rate was found increased in PD patients with $p<0.05$. Mean RR showed statistically significant reduction in PD patients $(822 \pm 115 \mathrm{~ms}$ in PD vs $983.2 \pm 116.8 \mathrm{~ms}$ in controls, $\mathrm{p}<0.05)$. The SDNN $(38.7 \pm 16.7 \mathrm{~ms}$ PD vs $51.36 \pm 21.65 \mathrm{~ms} \mathrm{C}, \mathrm{p}<0.01)$, rMSSD $(36.9 \pm 14.7 \mathrm{~ms}$ $\mathrm{PD}$ vs $43.1 \pm 8.6 \mathrm{~ms} \mathrm{C}, \mathrm{p}<0.05)$ were reduced in $\mathrm{PD}$ patients when compared to the control group.

Both LF and HF were lower in PD patients than in 
controls (LF: $332 . \pm 288.4 \mathrm{~ms}^{2} \mathrm{PD}$ vs $723.9 \pm 348.2 \mathrm{~ms}^{2}$ C; HF: $283.72 \pm 241.97 \mathrm{~ms}^{2}$ PD vs $530.54 \pm 226.5 \mathrm{~ms}^{2}$ $\mathrm{C}, \mathrm{p}<0.01$ ). No differences between $\mathrm{LF} / \mathrm{HF}$ ratio of PD and controls appeared.

Table 2. Characteristic of study groups, according to morphometric and comparison of time domain and spectral parameters of HRV

\begin{tabular}{ccc}
\hline Parameter & $\begin{array}{c}\text { Control } \\
\mathbf{n = 2 0}\end{array}$ & $\begin{array}{c}\text { PD } \\
\mathbf{n}=\mathbf{3 0}\end{array}$ \\
\hline Age (years) & $61.84 \pm 3.54$ & $63.2 \pm 3.47$ \\
BMI (Kg/m $\left.{ }^{2}\right)$ & $25.63 \pm 1.56$ & $24.44 \pm 1.74^{*}$ \\
UPDRS motor & - & $19.5 \pm 5.2$ \\
Fasting blood sugar & $91.1 \pm 22.12$ & $95.3 \pm 17.34$ \\
(mg/dl) & & \\
Total cholesterol (mg/dl) & $196.6 \pm 29.6$ & $185.4 \pm 21.33$ \\
HDL-cholesterol (mg/dl) & $47.2 \pm 3.1$ & $41.34 \pm 3.53^{*}$ \\
Triglyceride (mg/dl) & $158.2 \pm 14.96$ & $162.5 \pm 32.32$ \\
Vitamin B12 (pg/ml) & $581.9 \pm 108.4$ & $597.75 \pm 149.5$ \\
Acid folic (ng/ml) & $9.27 \pm 2.19$ & $9.34 \pm 2.31$ \\
Mean SBP (mmHg) & $131.86 \pm 20.5$ & $129.6 \pm 14.87$ \\
Mean DBP (mmHg) & $69.21 \pm 8.01$ & $71.17 \pm 9.17$ \\
HR (b/min) & $68.81 \pm 8.96$ & $73.31 \pm 9.82^{*}$ \\
Mean RR (ms) & $983.2 \pm 116.8$ & $822 \pm 115^{*}$ \\
SDNN (ms) & $51.36 \pm 21.65$ & $38.7 \pm 16.7 * *$ \\
rMSSD (ms) & $43.1 \pm 8.6$ & $36.9 \pm 14.7^{*}$ \\
pNN50 (\%) & $16.09 \pm 4.6$ & $12.41 \pm 4.8^{*}$ \\
LF (ms $\left.{ }^{2}\right)$ & $723.9 \pm 348.2$ & $332 . \pm 288.4^{* *}$ \\
HF (ms $\left.{ }^{2}\right)$ & $530.54 \pm 226$. & $283.72 \pm 241.97^{*}$ \\
& 5 & $*$ \\
LF/HF & $1.59 \pm 0.37$ & $1.79 \pm 0.97$ \\
\hline
\end{tabular}

BMI - body mass index, UPDRS- Unified Parkinson's Disease Rating Scale, MMSE- Mini-Mental Status Examination, BDI- Beck depression inventory

SBP - systolic blood pressure, DBP - diastolic blood pressure, MAP-mean arterial pressure, PP- pulse pressure, HR- heart rate

Mean RR, SDNN, rMSSD, pNN50, LF, HF, LF/HF: explanation in method section.

Data: expressed as means \pm standard deviation;

$*_{-} \mathrm{P}<0.05 ; * *_{-} \mathrm{P}<0.01$ for difference between controls and $\mathrm{PD}$

The results of Pearson correlation test, for a confidence interval of $95 \%$ indicate a significant inverse correlation between the age and LF values $(\mathrm{r}=$ - 0.41, $\mathrm{p}<0.02)$ and triglycerides with HF $(\mathrm{r}=-0.36$, $\mathrm{p}<0.05)$. Also HF was correlated with BDI score $(\mathrm{r}=0.43, \mathrm{p}<0.001)$.

Sensory nerve action potential in median nerve was reduced in PD patients: $9.81 \pm 5.2 \mu \mathrm{V}$ vs $11.53 \pm 5.5 \mu \mathrm{V}$ in controls, but we didn't find any statistical significance (Table 3). For median motor nerve parameters didn't show any differences between the study groups. The results of sural nerve exploration show that the sensitive potential amplitude was $8.16 \pm 2.36 \mu \mathrm{V}$ vs $10.95 \pm 2.73 \mu \mathrm{V}$ for the control group, difference with high statistical significance $(p<0.01)$. No differences in sNCV of sural nerve, $\mathrm{mNCV}$ of tibial or peroneal nerves between PD patients and controls appeared. Also we found differences between CMAP of tibial nerve in PD $3.72 \pm 1.73 \mu \mathrm{V}$ and controls $5.34 \pm 1.71 \mu \mathrm{V}$ with $\mathrm{p}<0.01$.

Table 3. Results of neuroelectrophysiological examination on controls and PD patients

\begin{tabular}{ccc}
\hline Parameter & $\begin{array}{c}\text { Control } \\
\mathbf{n}=\mathbf{2 0}\end{array}$ & $\begin{array}{c}\text { PD } \\
\mathbf{n}=\mathbf{3 0}\end{array}$ \\
Median & & \\
CMAP $(\mu \mathrm{V})$ & $7.23 \pm 2.2$ & $6.8 \pm 2.6$ \\
mNCV $(\mathrm{m} / \mathrm{s})$ & $48.3 \pm 3.2$ & $48 \pm 3.23$ \\
MDL $(\mathrm{ms})$ & $2.92 \pm 1.5$ & $2.82 \pm 1.5$ \\
SNAP $(\mu \mathrm{V})$ & $11.53 \pm 5.5$ & $9.81 \pm 5.2$ \\
SL $(\mathrm{ms})$ & $1.84 \pm 0.01$ & $1.9 \pm 0.01$ \\
sNCV $(\mathrm{m} / \mathrm{s})$ & $50.3 \pm 6.4$ & $48.73 \pm 6.5$ \\
Sural & & \\
SNAP $(\mu \mathrm{V})$ & $10.95 \pm 2.73$ & $8.16 \pm 2.36^{* *}$ \\
SL $(\mathrm{ms})$ & $3.4 \pm 0.1$ & $3.5 \pm 0.1$ \\
sNCV $(\mathrm{m} / \mathrm{s})$ & $46.28 \pm 6.29$ & $45.8 \pm 6.5$ \\
Tibial & & \\
CMAP $(\mu \mathrm{V})$ & $5.34 \pm 1.71$ & $3.72 \pm 1.73 * *$ \\
mNCV $(\mathrm{m} / \mathrm{s})$ & $43.71 \pm 4.15$ & $43.78 \pm 4.6$ \\
MDL $(\mathrm{ms})$ & $4.32 \pm 1.2$ & $5.4 \pm 1.21^{*}$ \\
Peroneal & & \\
CMAP $(\mu \mathrm{V})$ & $4.15 \pm 1.2$ & $3.9 \pm 1.6$ \\
mNCV $(\mathrm{m} / \mathrm{s})$ & $48.41 \pm 2.28$ & $46.15 \pm 5.21$ \\
MDL $(\mathrm{ms})$ & $2.93 \pm 1.21$ & $3.36 \pm 1.33$
\end{tabular}

SL-sensitive latency, SNAP-sensory nerve action potential, sNCV - sensory nerve conduction velocity, MDL- motor distal latency, CMAP- compound motor action potential, mNCV - motor nerve conduction velocity.

$*_{-} \mathrm{P}<0.05$; ** $-\mathrm{P}<0.01$ for difference between controls and $\mathrm{PD}$

The results of Pearson correlation test, for a confidence interval of $95 \%$ indicate a significant inverse correlation between age and the sensory nerve conduction velocity value of the sural nerve $(\mathrm{r}=-0.47$, $\mathrm{p}<0.05$ ).

\section{Discussion}

$\mathrm{PD}$ is a neurodegenerative disorder, with an average age at onset of about 60 years and with the aging of the population it is anticipated that the prevalence of PD will increase dramatically in the coming decades. Recent studies suggest that PD may start in the gastrointestinal tract. According to the 'Braak's' hypothesis, protein aggregation in enteric neurons 
spreads to the brain through the dorsal motor nucleus of the vagus (10). Pre-symptomatic PD patients had protein aggregation in the peripheral nervous system but not in the central nervous system (1). Protein aggregation ascended into the central nervous system and correlated with the development of motor dysfunction. Braak had postulated that the early Stages 1 to 3 of disease develop in patients who have not yet been diagnosed with classical motor symptoms of disease. Motor symptoms start to develop in Stage 3, when pathology has spread to the substantia nigra. Cognitive problems and dementia would occur when the neocortex becomes affected in Stages 5 and 6.

Alpha synuclein deposits have been found in the autonomic nervous system, in sensory and motor peripheral nerves $(12,23)$. Existing reports indicate that autonomic nervous system dysfunction occurs in the very early stages of PD. Evidence shows that both parasympathetic and sympathetic functions are impaired in PD (24).

123I-MIBG myocardial

scintigraphy

(Metaiodobenzylguanidine-a physiological analogue of noradrenaline) is clinically used to evaluate myocardial sympathetic nerve damage in PD. Reduced uptake of 123I-MIBG is considered to reflect cardiac sympathetic denervation, which precedes the neuronal loss of the paravertebral sympathetic ganglia, suggesting distal-dominant degeneration (25).

Patients with PD have shown suppressed heart rate responses to deep breathing indicating parasympathetic dysfunction (26). PD patients have low supine blood pressure levels and orthostatic hypotension is frequent because degeneration of sympathetic neurons is found (27). That can increase susceptibility to falls in PD patients.

HRV is widely accepted non-invasive, providing information on sympathetic and parasympathetic modulation of the sinus node. HF is regard as a marker of cardiac vagal activity. LF represents the sympathetic activity with vagal modulation. Goldstein et al, 2011, suggests that LF power should be seen as an index of baroreflex function rather than cardiac sympathetic tone (28). SDNN, pNN50\%, time domain indicator of the HRV, represents the activity of the vagal nerve.

In our study the analysis of HRV demonstrated that both parasympathetic and sympathetic nerve functions were impaired in PD patients because the measures of SDNN, pNN50\%, HF and $\mathrm{LH}$ were significantly lower than in controls. No differences between LF/HF ratio of PD and controls appeared. Our results agree with other studies demonstrating that both branches of autonomic nervous system are affected early in the course of PD.

In PD recent clinical and pathological studies point to defects in function of the peripheral nervous system in patients with early onset PD due to parkin mutation (29). The occurrence of peripheral neuropathy in a genetic type of PD suggests that a link may be present between central and peripheral neuronal degeneration. Reichling and Levine, 2011, suggest that in PD we can saw a combination of peripheral afferent abnormalities with central pathological changes resulting in neuropathic pain. They suggest that it is the result of the same neurodegenerative processes, but occuring in the peripheral nervous system $(30,31)$.

In a longitudinal study of PD patients Toth et al., 2008 , found that over half of the patients tested had peripheral neuropathy (14). The severity of the PN correlated with increased exposure to L-DOPA. Blood testing showed that the PD patients with PN had increased serum levels of methylmalonic acid (14). They also suggested that treatment with cobalamin will prevent the formation of methylmalonic acid, and thus could help protect against PN during long term L-DOPA therapy (14).

Abnormalities in SNAP or CMAP amplitude and a conservation of the nervous conduction velocity reflect axonal loss or degeneration (22). The signs of demyelination are decrease of the nervous conduction velocities, decrease of the action potentials, and an extension of the distal latencies (22). In our study we found reduced SNAP of sural nerve in PD patients. Therefore we concluded that the neuropathy in PD patients was mostly of a sensory axonal-predominant type. Our PD patients had normal levels of cobalamin suggesting that the presence of PN in early stages may be intrinsic to PD. PN may contribute to distal limb weakness, pain and risk of falling. A possible limitation of our study is the presence of a small number of subjects this decreases the statistical power to detect differences between the groups. Therefore, more research and longer observation of a larger group of patients are needed, including the most advanced form of Parkinson's disease. 


\section{Conclusions}

PD causes dysfunction of autonomic cardiovascular regulation and peripheral nerve involvement especially axonal neuropathy even in early stage of the disease. The neurodegeneration process occurs both in the central and peripheral nervous systems so we can consider that PD is a multi-systemic disorder. Future work will seek to clarify pathophysiological mechanisms inducing the peripheral nerve damage seen in idiopathic Parkinson's disease.

Conflict of Interest Statement: The Authors declare that they have no conflict of interests.

\section{References:}

1. Braak H, Rub U, Gai WP, Del Tredici K. Idiopathic Parkinson's disease: possible routes by which vulnerable neuronal types may be subject to neuroinvasion by an unknown pathogen. J Neural Transm. 2003;110:517-536

2. Zarow C, Lyness SA, Mortimer JA, Chui HC. Neuronal loss is greater in the locus coeruleus than nucleus basalis and substantia nigra in Alzheimer and Parkinson diseases. Arch. Neurol. 2003; 60:337-341

3. Marey-Semper I, Gelman M, Levi-Strauss M. A selective toxicity toward cultured mesencephalic dopaminergic neurons is induced by the synergistic effects of energetic metabolism impairment and NMDA receptor activation. J Neurosci. 1995; 15:5912-5918

4. De Erausquin A, Costa E, Hanbauer I. Calcium homeostasis, free radical formation and trophic factor dependence mechanisms in Parkinson's disease. Pharmacol.. 1994; 46: 467-482

5. Olanow C, Stern M, Sethi K. The scientific and clinical basis for the treatment of Parkinson disease. Neurology. 2009; 72 (4S): S1-S136. doi: 10.1212/WNL.0b013e3181a1d44c

6. Boveris A, Navarro A. Brain mitochondrial dysfunction in aging. IUBMB Life. 2008; 60: 308-314. doi: 10.1002/iub.46

7. Dawson TM, Dawson VL. Molecular pathways of neurodegeneration in Parkinson's disease. Science. 2003; 302:819-822

8. Hashimoto M, Rockenstein E, Crews L, Masliah E. Role of protein aggregation I mitochondrial dysfunction and neurodegeneration in Alzheimer's and Parkinson's diseases. Neuromolecular Med. 2003; 4:21-36
9. Huang Y, Cheung L, Rowe D, Halliday G. Genetic contributions to Parkinson's disease. Brain Research Reviews. 2004; 46:44-70

10. Braak H, Del Tredici K. Neuroanatomy and pathology of sporadic Parkinson's disease. Adv Anat Embryol Cell Biol.2009; 201:1-119

11. Haapaniemi TH, Pursiainen V, Korpelainen JT, Huikuri HV, Sotaniemi KA, Myllylä VV . Ambulatory ECG and analysis of heart rate variability in Parkinson's disease. J Neurol Neurosurg Psychiatry,.2001; 70:305-310

12. $\mathrm{Mu} \mathrm{L}$, Sobotka S, Chen J, Su H, Sanders I, Nyirenda T, et al. Parkinson disease affects peripheral sensory nerves in the pharynx. J Neuropathol Exp Neurol.. 2013; 72:614623. doi: 10.1097/NEN.0b013e3182965886

13. Ceravolo R, Cossu G, Bandettini di Poggio M, Santoro L, Barone P, Zibetti M, et al. Neuropathy and levodopa in Parkinson's disease: evidence from a multicenter study. Mov Disord.. 2013; 28:1391-1397. doi: 10.1002/mds.25585

14. Toth C, Brown MS, Furtado S, Suchowersky O, Zochodne D. Neuropathy as a potential complication of levodopa use in Parkinson's disease. Mov Disord.. 2008; 23:1850-1859. doi: 10.1002/mds. 22137

15. Hughes AJ, Daniel SE, Kilford L, Lees AJ. Accuracy of clinical diagnosis of idiopathic Parkinson's disease: a clinico-pathological study of 100 cases. J Neurol Neurosurg Psychiatry. 1993; 56:938-939

16. Hoehn MM, Yahr MD. Parkinsonism: onset, progression and mortality. Neurology.1967;17: 427- 442

17. Fahn S, Elton RL, and the members of the UPDRS Development Committee. UPDRS: unified Parkinson's disease rating scale in recent development in Parkinson's disease. In: Fahn S, Marsden CD, Calne DB, et al, eds. Macmillan Healthcare Information, Florham Park: Macmillan. 1987; 2:153-164

18. Constantinescu V, Matei D, Costache V, et al. Linear and nonlinear parameters of heart rate variability in ischemic stroke patients. Neurol Neurochir Pol. 2018;52(2):194-206 https://doi.org/10.1016/j.pjnns.2017.10.002

19. Constantinescu V, Matei D, Cuciureanu D, et al. Cortical modulation of cardiac autonomic activity in ischemic stroke patients.Acta Neurol Belg. 2016;116(4):473-480 
https://doi.org/10.1007/s13760-016-0640-3

20. Andritoi, Doru; Matei, Dana; Luca, Catalina; et al. Preliminary Study of HR Analysis on Patients Recovering after Stroke. Conference: 9th International Symposium on Advanced Topics in Electrical Engineering (ATEE) Location: Univ Politehnica Bucharest, Fac Elect Engn, Bucharest, ROMANIA, 2015, pages: 23-26.

21. Task Force of the European Society of Cardiology and the North American Society of Pacing and Electrophysiology. Heart rate variability: standards of measurement, physiological interpretation, and clinical use. Circulation. 1996; 93: 1043-1065

22. McLeod JG. Investigation of peripheral neuropathy. J Neurol Neurosurg Psych.1995; 58:274-283

23. Donadio V, Incensi A, Leta V, Giannoccaro MP, Scaglione C, Martinelli P, et al. Skin nerve $\alpha$ synuclein deposits: a biomarker for idiopathic Parkinson disease. Neurology. 2014; 82:13621369. doi: 10.1212/WNL.0000000000000316

24. Adhiyaman V, Hobson P, Meara RJ. Central and peripheral autonomic integrity in Parkinson's disease. Age Ageing. 2008; 37:578-581. doi: 10.1093/ageing/afn149

25. Orimo S, Takahashi A, Uchihara T, Mori F, Kakita A, Wakabayashi K, Takahashi H. Degeneration of cardiac sympathetic nerve begins in the early disease process of Parkinson's disease. Brain Pathology,.2007; 17:24-30

26. Kallio M, Haapaniemi T, Turkka J, Suominen K, Tolonen U, Sotaniemi K, Heikkilä VP, Myllylä $\mathrm{V}$. Heart rate variability in patients with untreated Parkinson's disease. Eur J Neurol. 2000; 7:667672

27. Jain S, Goldstein

DS.

Cardiovascular dysautonomia in Parkinson

disease: from pathophysiology to pathogenesis. Neurobiol Dis.. 2012; 46:572-580. doi: 10.1016/j.nbd.2011.10.025.

28. Goldstein DS, Bentho O, Park M-Y, Sharabi Y. Low-frequency power of heart rate variability is not a measure of cardiac sympathetic tone but may be a measure of modulation of cardiac autonomic outflows by baroreflexes. Exp Physiol. 2011; 96:1255-1261. doi: 10.1113/expphysiol.2010.056259

29. Ohsawa Y, Kurokawa K, Sonoo M, Yamada H, Hemmi S, Iwatsuki K, et al. Reduced amplitude of the sural nerve sensory action potential in PARK2 patients. Neurology. 2005; 65:459-462

30. Reichling DB, Levine JD. Pain and death: Neurodegenerative disease mechanism in the nociceptor. Ann Neurol. 2011; 69:13-21. doi: 10.1002/ana.22351

31. Munteanu C. - Cell biology considerations in Spinal Cord Injury - Review Balneo Research Journal. 2017; 8(3):136-151 DOI 10.12680/balneo.2017.149 